\title{
Twist-4 Matrix Elements of the Nucleon from Recent DIS Data at CERN and SLAC
}

\author{
S. Choi ${ }^{1}$, T. Hatsuda ${ }^{2}$, Y. Koike ${ }^{3}$ and Su H. Lee ${ }^{1,2}$ \\ ${ }^{1}$ Physics Department, Yonsei University, Seoul 120-749, Korea \\ ${ }^{2}$ Physics Department, FM-15, University of Washington, Seattle, WA 98195, USA \\ ${ }^{3}$ National Superconducting Cyclotron Laboratory, Michigan State University, \\ East Lansing, MI 48824-1321, USA
}

\begin{abstract}
We analyse the recent precision measurements of the lepton-hadron deep inelastic scattering at CERN and SLAC to extract model independent constraints among the nucleon matrix elements of the twist-4 operators. We also study a parameterization of these matrix elements and point out the possibility that the matrix elements of the quark-gluon mixed operator has a negative value of the order of $-(400 \pm 100 \mathrm{MeV})^{2}$ at $5 \mathrm{GeV}^{2}$ renormalization scale.
\end{abstract}




\section{Introduction}

Measurements of the lepton-hadron deep inelastic scattering(DIS) remain to be the cornerstone of various QCD tests, ranging from precise determination of $\Lambda_{Q C D}$ to the knowledge of the structure functions necessary to calculate cross sections for hard scattering processes. (For the recent review, see [1].)

Recent precision DIS data at CERN [2, 3, 4] and at SLAC [5] also provide us with a fruitful byproduct, i.e. the estimate of higher twist effects in the spin averaged structure functions $\left(F_{2}\right.$ and $\left.F_{L}\right)$. The twist-4 part of these structure functions is defined through

$$
F_{2, L}\left(x, Q^{2}\right)=F_{2, L}^{\tau=2}\left(x, Q^{2}\right)+\frac{1}{Q^{2}} F_{2, L}^{\tau=4}\left(x, Q^{2}\right)
$$

where the target mass corrections [6] are taken into account in the twist-2 part $F_{2, L}^{\tau=2}$.

In terms of the operator product expansion (OPE), the four-quark operators $\left(\bar{\Psi} \Gamma_{\mu_{1}} \Psi \bar{\Psi} \Gamma_{\mu_{2}} \Psi\right)$ and the quark-gluon mixed operator $\left(\bar{\Psi}\left\{D_{\mu_{1}},{ }^{*} F_{\mu_{2} \alpha}\right\} \Gamma^{\alpha} \Psi\right)$ contribute to $F_{2, L}^{\tau=4}$ [7, 8, 9, 10]. The twist-2 part $F_{2, L}^{\tau=2}$ is known to give a parton distribution (i.e. the single particle property of quarks and gluons in the nucleon), while the matrix elements of the twist-4 operators are the measure of the correlation of quarks and gluons in the nucleon.

Such new information has wide applications in QCD: first of all it gives a detailed knowledge of the nucleon structure and gives a stringent test of the various models of the nucleon. Secondly, these twist-4 matrix elements are useful to analyse the higher twist effects in other high energy processes such as the neutrino induced reaction and the Drell-Yan processes [11]. Thirdly, the twist-4 matrix elements are essential to study the propagation of hadrons in nuclear medium as is shown in the framework of the QCD sum rules [12].

At present, an unambiguous determination of the magnitude of the twist-4 matrix elements is not available. However, the recent NMC data [2, 3] together with the SLAC [5] and BCDMS [4] data give us a useful constraint among the twist-4 matrix elements. In this paper, we will first examine such constraints in a model independent way. Then, we will introduce a parameterization to satisfy the constraints and point out that the quark-gluon mixed operator at $5 \mathrm{GeV}^{2}$ scale has a sizable nucleon matrix element. 


\section{Operator Product Expansion}

The spin-2, twist-4 contribution to the spin-averaged forward amplitude of the electromagnetic current $j_{\mu}^{e m}$ can be written as [8, 9]

$$
\begin{aligned}
T_{\mu \nu} & =i \int d^{4} \xi e^{i q \xi}\left\langle T j_{\mu}^{e m}(\xi) j_{\nu}^{e m}(0)\right\rangle_{N} \\
& \rightarrow d_{\mu \nu} \frac{1}{x^{2} Q^{2}}\left(A^{1}+\frac{5}{8} A^{2}+\frac{1}{16} A^{g}\right)+e_{\mu \nu} \frac{1}{x^{2} Q^{2}}\left(\frac{1}{4} A^{2}-\frac{3}{8} A^{g}\right),
\end{aligned}
$$

where the polarization tensors are defined as $e_{\mu \nu}=g_{\mu \nu}-q_{\mu} q_{\nu} / q^{2}$ and $d_{\mu \nu}=-p_{\mu} p_{\nu} q^{2} /(p \cdot q)^{2}+$ $\left(p_{\mu} q_{\nu}+p_{\nu} q_{\mu}\right) / p \cdot q-g_{\mu \nu}$ with $Q^{2}=-q^{2} .\left(p^{\mu}\right.$ is a 4-momentum of the nucleon with $p^{2}=M_{N}^{2}$.) $A^{1,2, g}$ are the spin-averaged nucleon matrix elements of the spin-2, twist-4 operators:

$$
\left\langle\mathcal{O}_{\alpha \beta}^{k}\right\rangle=\left(p_{\alpha} p_{\beta}-\frac{1}{4} M_{N}^{2} g_{\alpha \beta}\right) A^{k},
$$

with

$$
\begin{aligned}
\mathcal{O}_{\alpha \beta}^{1} & =g^{2}\left(\bar{q} \gamma_{\alpha} \gamma_{5} \mathcal{Q} t^{a} q\right)\left(\bar{q} \gamma_{\beta} \gamma_{5} \mathcal{Q} t^{a} q\right), \\
\mathcal{O}_{\alpha \beta}^{2} & =g^{2}\left(\bar{q} \gamma_{\alpha} \mathcal{Q}^{2} t^{a} q\right)\left(\bar{q} \gamma_{\beta} t^{a} q\right) \\
\mathcal{O}_{\alpha \beta}^{g} & =i g\left(\bar{q}\left\{D_{\alpha},{ }^{*} F_{\beta \mu}\right\} \gamma^{\mu} \gamma_{5} \mathcal{Q}^{2} q\right)
\end{aligned}
$$

Here, the operators are assumed to be symmetric and traceless with respect to the Lorentz indices: $\mathcal{O}_{\alpha \beta} \rightarrow \frac{1}{2}\left(\mathcal{O}_{\alpha \beta}+\mathcal{O}_{\beta \alpha}\right)-\frac{1}{4} g_{\alpha \beta} \mathcal{O}_{\gamma \gamma}$. $\mathcal{Q}$ is the flavor $S U(2)$ charge matrix and $t^{a}$ are the generators of the color $\mathrm{SU}(3)$ normalized to $\operatorname{tr}\left(t^{a}\right)^{2}=1 / 2 . F_{\alpha \beta}=F_{\alpha \beta}^{a} t^{a}$, and the dual field strength is defined as ${ }^{*} F_{\alpha \beta}=\epsilon_{\alpha \beta \gamma \delta} F^{\gamma \delta}$ with $\epsilon_{0123}=1$. Here we have neglected the twist4 operators proportional to the current quark masses. A typical diagram which generates

$\mathcal{O}^{1}$ is given in Fig. 1(a), and that for $\mathcal{O}^{g}, \mathcal{O}^{2}$ is given in Fig. 1(b). If one writes eq. (2) as $T=2 M / x^{2} Q^{2}$, twist-4 matrix elements and the twist-4 structure functions are related as

$$
M_{2, L}\left(Q^{2}\right)=\int_{0}^{1} d x F_{2, L}^{\tau=4}\left(x, Q^{2}\right) .
$$

\section{Experimental data}

Structure Function $F_{2}(x)$ 
The experimental data of $F_{2}^{\tau=4}(x)$ have been analyzed by introducing the following unknown function $C(x)$

$$
F_{2}^{\tau=4}=C(x) F_{2}^{L T}\left(x, Q^{2}\right)
$$

where $F_{2}^{L T}\left(x, Q^{2}\right)$ denotes the leading-twist structure function with the target mass correction 6 .

$C(x)$ has been extracted for the hydrogen and deuterium target in ref. [13] by using the BCDMS data and the SLAC data taken in the kinematic region $0.07<x<0.75$ and $0.5<Q^{2}<260 \mathrm{GeV}^{2}$. We have carried out $\chi^{2}$ fitting of the proton data $C_{p}(x)$ (given in Table 2 of [13) by

$$
C(x)=a_{0}+a_{1} x+a_{2} x^{2}+a_{3} x^{3}+a_{4} x^{4},
$$

and we get $a_{0}=-0.28, a_{1}=3.45, a_{2}=-17.13, a_{3}=31.64$, and $a_{4}=-14.95$.

One can also extract $C_{n}(x)$ by combining hydrogen and deuterium data in [13]. The result, however, has large error bars. On the other hand, the NMC group recently published better statistics data for $C_{p}(x)-C_{n}(x)$ (but not for $C_{p}(x)$ and $C_{n}(x)$ separately) which is a combination of NMC, SLAC and BCDMS data [3]. The NMC group analyzed the ratio $F_{2}^{n} / F_{n}^{p}$ in the kinematic range $0.07<x<0.75$ and $0.8<Q^{2}<75 \mathrm{GeV}^{2}$. This ratio is independent of the spectrometer acceptance and normalization and gives a reliable estimate of $C_{p}(x)-C_{n}(x)$ from the following relation,

$$
\frac{F_{2}^{n}}{F_{2}^{p}}=\left(\frac{F_{2}^{n}}{F_{2}^{p}}\right)^{L T}\left(1-\frac{C_{p}(x)-C_{n}(x)}{Q^{2}}\right) .
$$

By combining this data with that of the proton in ref. [13] and fitting the resulting values for $C_{n}(x)$ with the same polynomial in eq. ([7), we obtain the following values for the coefficients; $a_{0}=-0.28, a_{1}=3.12, a_{2}=-11.01, a_{3}=16.51$, and $a_{4}=-2.40$. We checked that different set of fittings fall well within the estimated errors of the following results.

From our fit of $C_{p}(x)$ and $C_{n}(x)$, the integrated structure function at $Q^{2}=5 \mathrm{GeV}^{2}$ (which is a typical scale where the twist-4 effect is extracted) reads

$$
\begin{aligned}
\int_{0}^{1} F_{2}^{\tau=4} d x & =\frac{1}{2}\left(A^{1}+\frac{5}{8} A^{2}+\frac{1}{16} A^{g}\right) \\
& =\int_{0}^{1} C(x) F_{2}^{L T}(x) d x=\left\{\begin{array}{lll}
0.005 \pm 0.004 & \mathrm{GeV}^{2} \text { (proton) } \\
0.011 \pm 0.004 & \mathrm{GeV}^{2} \text { (neutron) }
\end{array} .\right.
\end{aligned}
$$


The errors come from unavailability of $C(x)$ for $x>0.75$ and $x<0.07$. Here we have used the leading order (LO) structure function of Glück-Reya-Vogt 14 for $F_{2}^{L T}$. At $Q^{2} \sim 5$ $\mathrm{GeV}^{2}$, the difference between the LO and the higher order $(\mathrm{HO})$ distribution functions are not significant after the $x$-integration. ${ }^{1}$

\section{Longitudinal Structure Function $F_{L}(x)$}

The higher twist effect in the longitudinal structure function is obtained by the ratio between the longitudinal and transverse cross sections $R=\sigma_{L} / \sigma_{T}$. This ratio is especially sensitive to the higher twist contribution because the lowest twist effect to $F_{L}$ is of order $\alpha_{s}$. Note that only diagrams such as given in Fig. 1(b) contribute to $F_{L}$. In this case, the twist-4 analysis using the transverse basis provides us with an intuitive picture [10], in which the higher twist effects can be interpreted in terms of the intrinsic transverse momentum of partons: $F_{L}^{\tau=4}(x)=4 \int d^{2} k_{T} k_{T}^{2} f\left(x, k_{T}^{2}\right)$, where $f\left(x, k_{T}^{2}\right)$ denotes a structure function for quarks with the momentum fraction $x$ and the transverse momentum $k_{T}$.

Motivated by this, the SLAC data [0] was analysed in ref. [15] by introducing a typical scale for the transverse momentum of the parton $\kappa$, 2ّ

$$
F_{L}^{\tau=4}\left(x, Q^{2}\right)=8 \kappa^{2} F_{2}^{L T}\left(x, Q^{2}\right)
$$

By using the leading and next-to-leading order structure function for $F_{2}^{L T}$, the SLAC data can be fitted by

$$
\kappa^{2}=0.03 \pm 0.01 \mathrm{GeV}^{2},
$$

in the range $0.2<x<0.6$ [15, [16]. An indirect experimental justification of eq. (10]) is that $R$ is independent of targets [2, 5]. If the twist-4 contribution to $F_{L}$ were not proportional to $F_{2}$, the twist- 4 contribution to $R$ would depend on the targets. Using the above fit, we obtain $\left(\text { at } Q^{2}=5 \mathrm{GeV}^{2}\right)^{3}$

$$
\int_{0}^{1} F_{L}^{\tau=4} d x=\frac{1}{2}\left(\frac{1}{4} A^{2}-\frac{3}{8} A^{g}\right)
$$

\footnotetext{
1 Although this phenomenological parton distribution function might contain the effect of the power corrections, this portion will be $O\left(1 / Q^{4}\right)$ and thus irrelevant in the twist-4 part of $F_{2}$ in eq. (1).

${ }^{2}$ This will be an important guide for our parameterization in section 3 .

${ }^{3}$ Here we have again used the LO structure function of ref. [14].
} 


$$
=\int_{0}^{1} 8 \kappa^{2} F_{2}^{L T}(x) d x=\left\{\begin{array}{lll}
0.035 \pm 0.012 & \mathrm{GeV}^{2} & \text { (proton) } \\
0.023 \pm 0.008 & \mathrm{GeV}^{2} & \text { (neutron) }
\end{array} .\right.
$$

As is clear from this expression, the difference between the proton and the neutron comes only from the difference in $\int F_{2}^{L T} d x$.

\section{Constraints on $A^{1,2, g}$}

The experimental data for $F_{L}^{\tau=4}$ (eq. (12)) is 2-7 times larger than those for $F_{2}^{\tau=4}$ (eq. (9)). Since both $A^{1}$ and $A^{2}$ are the matrix elements of the four-quark operators, their absolute values are expected to be similar in magnitude. This together with eqs. (12) and (9) suggests that $A^{g}$ at $Q^{2}=5 \mathrm{GeV}^{2}$ takes large and negative value to reproduce $F_{2}$ and $F_{L}$ simultaneously. We will come back to this point in section 5 .

From eqs. (12) and (9), we can derive two constraints among $A^{1}, A^{2}$ and $A^{g}$ :

$$
\begin{aligned}
& A^{1}=-A^{g}+\left\{\begin{array}{lll}
-0.165 \pm 0.061 & \mathrm{GeV}^{2} & \text { (proton) } \\
-0.093 \pm 0.041 & \mathrm{GeV}^{2} & \text { (neutron) }
\end{array}\right. \\
& A^{2}=\frac{3}{2} A^{g}+\left\{\begin{array}{lll}
0.280 \pm 0.096 & \mathrm{GeV}^{2} & \text { (proton) } \\
0.184 \pm 0.064 & \mathrm{GeV}^{2} & \text { (neutron) }
\end{array} .\right.
\end{aligned}
$$

The $A^{1}-A^{g}$ and $A^{2}-A^{g}$ relations with error bars are given as the bands in Fig. 2 . The figure shows that it is hard to find a solution where $A^{1,2, g}$ are all consistent with zero, which

clearly indicates sizable values of the twist-4 matrix elements. We note that as long as $A^{1}$, $A^{2}$ and $A^{g}$ do not take too different values among one another, the typical magnitude of them reads $0.1 \mathrm{GeV}^{2} \sim(300 \mathrm{MeV})^{2}$ and a negative value for $A^{g}$ is favored. (We will discuss this in detail in section 5.)

Fig. 2 gives an useful test of the various nucleon models: Any reliable models of the nucleon should be able to predict the matrix elements within the bands in Fig. 2. One should also note that twist- 4 data of $F_{3}(x)$, although it is not available now, will be particularly useful to obtain further constraints on $A^{1,2, g}$.

\section{Parameterization of the matrix elements}

Although Fig. 2 provides us with a model-independent constraint among the twist-4 matrix elements, it does not give any definite numbers for the matrix elements. In this section, we 
will further introduce a theoretical assumption to estimate the magnitude of $A^{1,2, g}$.

\section{The Bag Model}

The MIT bag model provides us with the simplest estimate of the twist-4 matrix elements. Jaffe and Soldate calculated $A^{1}$ and $A^{2}$ and found that $F_{2}^{\tau=4}$ in the model has an opposite sign from the data (see the footnote 15 of the latter reference in [9]). Shuryak and Veinstein [8] also discussed that models without correlation between quarks inside the nucleon cannot reproduce the data. Let's first generalize the MIT bag model parameterization to see whether one can remedy the problem encountered in [9].

The nucleon expectation values of any operators in eq. (四) can be obtained from the bag wave function as follows:

$$
A^{k}=\frac{2}{M_{N}} \int d^{3} x\left\langle\hat{p}\left|\mathcal{O}_{00}^{k}+\frac{1}{3} \mathcal{O}_{i i}^{k}\right| \hat{p}\right\rangle, \quad k=1,2, g
$$

where $|\hat{p}\rangle$ is the bag state made of three confined quarks. By using the explicit form of $|\hat{p}\rangle$, one obtains [9], $A^{1}=(2 / 3) f_{1} a-(16 / 9) f_{2} a$ and $A^{2}=2 f_{1} b+(16 / 9) f_{2} c$. Here the factors related to the color-spin-charge read $a=-16 / 9(-4 / 3), b=-4 / 3(-8 / 9)$ and $c=8 / 9(4 / 3)$ for the proton (neutron). $f_{1,2}$ is related to the spacial wave function of quarks: A simple estimate with the bag radius $1 \mathrm{fm}$ gives $f_{1}=0.0266 \times \alpha_{s}$ and $f_{2}=0.0042 \times \alpha_{s}$, which leads to $A^{1}=-0.018(-0.014) \times \alpha_{s} \mathrm{GeV}^{2}$ and $A^{2}=-0.064(-0.037) \times \alpha_{s} \mathrm{GeV}^{2}$ for the proton (neutron). $\alpha_{s}$ is the strong coupling constant and we adopt $\alpha_{s} \sim 0.5$. .

The mixed condensate can also be obtained from eq. (14) by assuming abelian electric and magnetic fields. The electric field vanishes locally within the bag, while the magnetic field together with the quark wave function in the bag gives

$$
A^{g}=\left\{\begin{array}{lll}
0.075 \times \alpha_{s} & \mathrm{GeV}^{2} & \text { (proton) } \\
0.113 \times \alpha_{s} & \mathrm{GeV}^{2} & \text { (neutron) } .
\end{array}\right.
$$

Here $\mathcal{O}_{00}^{g}$ has a dominant and positive contribution to $A^{g}$.

Adding all the contributions we finally obtain

$$
\int_{0}^{1} F_{2}^{\tau=4} d x=\left\{\begin{array}{lll}
-0.027 \times \alpha_{s} & \mathrm{GeV}^{2} & \text { (proton) } \\
-0.015 \times \alpha_{s} & \mathrm{GeV}^{2} & \text { (neutron) },
\end{array}\right.
$$

\footnotetext{
${ }^{4}$ Here it is not obvious whether one should use $\alpha_{s}$ at $Q^{2}=5 \mathrm{GeV}^{2}$ or something else. In this paper, we follow the argument in [9] to estimate an "effective" value $\alpha_{s} \sim 0.5$.
} 
and

$$
\int_{0}^{1} F_{L}^{\tau=4} d x=\left\{\begin{array}{lll}
-0.022 \times \alpha_{s} & \mathrm{GeV}^{2} & \text { (proton) } \\
-0.026 \times \alpha_{s} & \mathrm{GeV}^{2} & \text { (neutron) }
\end{array}\right.
$$

Comparing these with eqs. (9) and (12), one finds that the bag model gives incorrect signs although the absolute values are the right order of magnitude. The circle (proton) and the cross (neutron) in Fig. 2 denote the prediction of the bag model, which shows that the model is inconsistent with the current data.

One may get opposite signs for $A^{1}$ and $A^{2}$ by making $f_{2}$ comparable to $f_{1}$. However, for any reasonable form of the wave function, $f_{2}$ is much smaller than $f_{1}$ and in fact the bag model gives the most generous estimate. Diquark models give positive signs for the moments [17], but they do not fit the $x$ dependence of the structure function [5].

\section{A parameterization based on flavor structure}

Instead of introducing more sophisticated models of the nucleon, we now discuss a different kind of parameterization motivated by eq. (10). Let us first rewrite the matrix elements of the operators in eq. (四) by using the charge operator $\mathcal{Q}=\operatorname{diag} .\left(Q_{u}, Q_{d}\right)$,

$$
\begin{aligned}
& A_{p(n)}^{1}=Q_{u}^{2} K_{u(d)}^{1}+Q_{d}^{2} K_{d(u)}^{1}-\left(Q_{u}-Q_{d}\right)^{2} K_{u d}^{1} / 2 \\
& A_{p(n)}^{2}=Q_{u}^{2} K_{u(d)}^{2}+Q_{d}^{2} K_{d(u)}^{2} \\
& A_{p(n)}^{g}=Q_{u}^{2} K_{u(d)}^{g}+Q_{d}^{2} K_{d(u)}^{g}
\end{aligned}
$$

where $K$ 's are the matrix elements defined by

$$
\begin{aligned}
K_{u}^{i} & =\frac{2}{M^{2}}\left\langle\bar{u} \Gamma_{+}^{i} \Delta_{+}^{i} u\right\rangle_{p}, \quad i=1,2 \\
K_{u}^{g} & =\frac{2 i g}{M^{2}}\left\langle\bar{u}\left\{D_{+}{ }^{*} F_{+\mu}\right\} \gamma^{\mu} \gamma_{5} u\right\rangle_{p} \\
K_{u d}^{1} & =\frac{2}{M^{2}}\left\langle 2\left(\bar{u} \Gamma_{+}^{1} u\right)\left(\bar{d} \Gamma_{+}^{1} d\right)\right\rangle_{p} .
\end{aligned}
$$

Here, $\Gamma_{\alpha}^{1}=\gamma_{\alpha} \gamma_{5} t^{a}, \Gamma_{\alpha}^{2}=\gamma_{\alpha} t^{a}, \Gamma_{+}=\frac{1}{\sqrt{2}}\left(\Gamma_{0}+\Gamma_{3}\right)$ and $\Delta_{\alpha}^{i}=\bar{u} \Gamma_{\alpha}^{i} u+\bar{d} \Gamma_{\alpha}^{i} d$ is a flavor-singlet operator. The neutron matrix elements are obtained from those of the proton by the isospin symmetry and we have neglected the strangeness contribution to simplify the analysis. 
Noting that the flavor structure of $K_{d}^{1,2, g}$ and that of $K_{u}^{1,2, g}$ are governed by the $d$-quark and the $u$-quark respectively, we will introduce an ansatz in which the ratio $K_{d}^{1,2, g} / K_{u}^{1,2, g}$ is equal to the momentum fraction of the $d$ and $u$ quarks in the nucleon:

$$
K_{d}^{1,2, g} / K_{u}^{1,2, g} \simeq \int x(d(x)+\bar{d}(x)) d x / \int x(u(x)+\bar{u}(x)) d x \equiv \beta
$$

Here $u(x), d(x), \cdots$ are the usual twist-2 parton distribution functions. $\beta$ takes a value 0.476 at $Q^{2}=5 \mathrm{GeV}^{2}$. The analogous condition for $K_{d}^{2, g} / K_{u}^{2, g}$ in eq. (20) is a sufficient condition to satisfy eq. (10), which can be checked by substituting eq. (18) into eq. (12) and equating the charge operators in both sides. Thus essentially it does not bring any new constraints. On the other hand, the condition for $K_{d}^{1} / K_{u}^{1}$ is purely an ansatz: Although it is plausible from the point of view of the flavor-structure of the operator, it needs to be checked by a non-perturbative method in QCD.

With eq. (20), we can reduce the number of matrix elements from $6\left(A^{1,2, g}\right.$ for the proton and the neutron) to $4\left(K_{u}^{1,2, g}, K_{u d}^{1}\right)$. Although we have 4 experimental inputs, we cannot determine all of them uniquely since the ratio of the proton and neutron data for $F_{L}^{\tau=4}$ is automatically satisfied in our parameterization. Therefore, we will vary $K_{u d}^{1}$ and solve others as functions of $K_{u d}^{1}$. We will also limit the variation of $\left|K_{u d}^{1}\right|$ in the range between $\left|K_{d}^{1}\right|$ and $\left|K_{u}^{1}\right|$. (In fact, the difference between $\left(K_{u}^{1}, K_{d}^{1}\right)$ and $K_{u d}^{1}$ is only the flavor structure and QCD is flavor-blind, therefore these matrix elements should take the similar values in magnitude.) The resulting values in $\mathrm{GeV}^{2}$ unit are given in Table 1.

\begin{tabular}{|c|c|c|c|c|c|c|c|}
\hline$K_{u d}^{1}$ & $K_{u}^{1}$ & $K_{u}^{2}$ & $K_{u}^{g}$ & $K_{u d}^{1}$ & $K_{u}^{1}$ & $K_{u}^{2}$ & $K_{u}^{g}$ \\
\hline \hline$K_{d}^{1}$ & -0.173 & 0.203 & -0.238 & $-K_{u}^{1}$ & 0.083 & -0.181 & -0.494 \\
\hline$\left(K_{d}^{1}+K_{u}^{1}\right) / 2$ & -0.112 & 0.110 & -0.300 & $-\left(K_{d}^{1}+K_{u}^{1}\right) / 2$ & 0.112 & -0.225 & -0.523 \\
\hline$K_{u}^{1}$ & -0.083 & 0.066 & -0.329 & $-K_{d}^{1}$ & 0.173 & -0.318 & -0.585 \\
\hline
\end{tabular}

Table 1

Table 1 gives the following constraints on the possible range of $A^{g}$ at $5 \mathrm{GeV}^{2}$ scale:

$$
\begin{array}{ll}
-(540 \mathrm{MeV})^{2}<A^{g}<-(340 \mathrm{MeV})^{2} & \text { (proton) } \\
-(440 \mathrm{MeV})^{2}<A^{g}<-(280 \mathrm{MeV})^{2} \quad \text { (neutron) }
\end{array}
$$

which favor the region inside the parallelograms in Fig. 2. (Note that the results in the 
present parameterization are always confined inside the bands in Fig. 2 contrary to those of the bag model.)

Our analysis here suggests that:

1. As we have discussed in section 4, the matrix element of the quark-gluon mixed operator $A^{g}$ is relatively large compared to the four quark operators at $5 \mathrm{GeV}^{2}$ scale. The magnitude of the former is about $-(300-500 \mathrm{MeV})^{2}$ which is consistent with a typical hadronic scale. The sign and the magnitude of the matrix elements should be understood in a microscopic manner (either by lattice QCD or by non-perturbative nucleon models). To compare model calculations with our results in a quantitative manner, one needs to evolve $A^{1,2, g}$ from $5 \mathrm{GeV}^{2}$ scale to the typical hadronic scale. This requires further knowledge of the anomalous dimensions of the operators in eq. (田). Our result here is also relevant to the analysis of the QCD sum rules in the nuclear medium [12].

2. One can show that $A^{1}$ and $A^{2}$ have opposite signs from Table 1 . This causes a relatively strong cancellation in $F_{2}^{\tau=4}(x)$ providing with a reason for the large difference between the data on $F_{2}$ (eq. (9)) and on $F_{L}$ (eq. (12)).

S.H.L and T.H. were supported by U.S. Department of Energy under grant DE-FG0688ER40427. C. S. and S.H.L were partly supported by Yonsei University Faculty Research grant. Y. K. was supported by the US National Science Foundation under grant PHY9017077. One of the authors (Y. K.) thanks W.K. Tung for useful discussions. 


\section{References}

[1] D. von Harrach, Nucl. Phys. A546 (1992) 1c.

[2] P. Amaudruz et al. (New Muon Collaboration), Phys. Lett. 294B (1992) 120; Phys. Lett. 295B (1992) 159.

[3] P. Amaudruz et al. (New Muon Collaboration), Nucl. Phys. B371 (1992) 3.

[4] A. C. Benevenuti et al. (BCDMS Collaboration), Phys. Lett. B223 (1989) 485; B237 (1990) 592.

[5] L. W. Whitlow et al. Phys. Lett. 250B (1990) 193; Phys. Lett. 282B (1992) 475.

[6] O. Nachtmann, Nucl. Phys. B63 (1973) 237;

H. Georgi and H. Politzer, Phys. Rev. D14 (1976) 1829.

[7] H. D. Politzer, Nucl. Phys. B172 (1980) 349.

[8] E. V. Shuryak and A. I. Vainshtein, Phys. Lett. 105B (1981) 65, Nucl. Phys. B199 (1982) 451.

[9] R.L. Jaffe and M. Soldate, Phys. Lett. B105 (1981) 467, Phys. Rev. D26 (1982) 49.

[10] R. K. Ellis, W. Furmanski and R. Petronzio, Nucl. Phys. B207 (1982) 1; B212 (1983) 29.

[11] J. Qiu and G. Sterman, Nucl. Phys. B353 (1991) 105.

[12] T. Hatsuda and Su H. Lee, Phys. Rev. C46 (1992) R34; T. Hatsuda, Y. Koike and Su H. Lee, in preparation.

[13] M. Virchaux and A. Milsztajn, Phys. Lett. 274B (1992) 221.

[14] M. Glück, E. Reya and A. Vogt, Z. Phys. C48 (1990) 471.

[15] J. L. Miramontes, M. A. Miramontes and J. Sanchez Guillen, Phys. Rev. D40 (1989) 2184.

[16] J. Sanchex Guillen, J. L. Miramontes, M. Miramontes, G. Parente and O.A. Sampayo, Nucl. Phys. B353 (1991) 337.

[17] S. Ekelin and S. Fredriksson, Phys. Lett. 162B (1985) 373. 


\section{Figure Captions}

Fig. 1: Typical diagrams for the twist-4 contribution to the forward Compton amplitude: (a) the four quark contribution and (b) the quark-gluon mixed contribution.

Fig. 2: The twist-4 matrix elements $A_{1}$ and $A_{2}$ as a function of $A_{g}$ in the unit of $\mathrm{GeV}^{2}$. They are evaluated at the renormalization scale $\mu^{2}=5 \mathrm{GeV}^{2}$. The band in solid line (dashed line) is a region allowed by the experimental data for the proton (neutron). The circle (cross) is a prediction for the proton (neutron) in the MIT bag model. The region inside the parallelogram is allowed in the parameterization based on the flavor structure of the twist-4 operators. 